\title{
Coexistence of magnetic order and two-dimensional superconductivity at $\mathrm{LaAlO}_{3} / \mathrm{SrTiO}_{3}$ interfaces
}

\author{
Lu Li ${ }^{1}$, C. Richter ${ }^{2}$, J. Mannhart ${ }^{3}$ and R. C. Ashoori ${ }^{1 \star}$
}

\begin{abstract}
A two-dimensional electronic system forms at the interface between the band insulators ${ }^{1,2} \mathrm{LaAlO}_{3}$ and $\mathrm{SrTiO}_{3}$. Samples fabricated until now have been found to be either magnetic or superconducting, depending on growth conditions ${ }^{3,4}$. Combining high-resolution magnetic torque magnetometry and transport measurements, we report here magnetization measurements providing direct evidence of magnetic ordering of the two-dimensional electron liquid at the interface. The magnetic ordering exists from well below the superconducting transition to up to $200 \mathrm{~K}$, and is characterized by an in-plane magnetic moment. Surprisingly, despite the presence of this magnetic ordering, the interface superconducts below $120 \mathrm{mK}$. This is unusual because conventional superconductivity rarely exists in magnetically ordered metals ${ }^{5,6}$. Our results suggest that there is either phase separation or coexistence between magnetic and superconducting states. The coexistence scenario would point to an unconventional superconducting phase as the ground state.
\end{abstract}

Superconductivity and magnetic order are in general mutually exclusive phenomena. Nonetheless, the coexistence of magnetism and superconductivity has been suggested for finite-momentum pairing states ${ }^{5,6}$. Coexistence of magnetism and superconductivity has been reported in a few three-dimensional superconducting systems ${ }^{7-9}$, such as $\mathrm{RuSr}_{2} \mathrm{GdCu}_{2} \mathrm{O}_{8}$ and $\mathrm{UGe}_{2}$. The question remains if such coexistence can occur in a two-dimensional electronic system. An intriguing candidate is the interface between the two band insulators $\mathrm{LaAlO}_{3}$ (LAO) and $\mathrm{SrTiO}_{3}$ (STO). At their ntype interface a conducting two-dimensional electron liquid is generated. Moreover, the LAO/STO interface was also reported to have a two-dimensional superconducting ground state ${ }^{3}$.

For this system, magnetic ordering was suggested in ref. 4, the authors of which deduced the presence of magnetic scattering centres from the temperature dependence of the interface resistance $R$ and a hysteresis of $R$ during the sweep of magnetic field $H$. Different magnetotransport studies indicate an antiferromagnetic order ${ }^{10}$ or a non-uniform field-induced magnetization and strong magnetic anisotropy ${ }^{11}$. Recently, it was found that, at both chemically treated STO bulk and LAO/STO interfaces, charges are electronically phase separated into regions containing a quasi-two-dimensional electron-gas phase, a ferromagnetic phase persisting above room temperature or a diamagnetic/paramagnetic phase ${ }^{12}$ below $60 \mathrm{~K}$. On the theoretical side, electronic-structure calculations yield complicated pictures for the magnetism at the interface layers ${ }^{13-16}$. Specifically, the calculations do not support magnetically ordered moments at the interface of an LAO/STO bilayer covered by vacuum $^{17}$. Consequently, any observed magnetism must originate from strong electronic correlations.

Coexistence of magnetism and superconductivity has not been reported at the LAO/STO interfaces. The ground state was found to be controlled by growth conditions, carrier concentration ${ }^{18}$ and external magnetic field ${ }^{19}$. These experimental observations based on transport properties suggest that the two phenomena do not coexist (see, for example, Fig. 16 of ref. 18).

To clarify this issue, we have grown LAO/STO interfaces, measured their superconducting properties by transport measurements and then applied cantilever-based torque magnetometry as an extremely sensitive and direct method to measure a possible magnetic moment $m$ of the sample.

Torque magnetometry directly determines $m$ by measuring the torque $\boldsymbol{\tau}$ of the sample mounted on a cantilever in an external magnetic field $H$. As the torque is given by $\boldsymbol{\tau}=\mathbf{m} \times \mathbf{B}$, the method detects the component of $\mathbf{m}$ oriented perpendicular to $\mathbf{B}$, the magnetic field flux density. Owing to its great sensitivity, this method has been applied to determine the magnetic susceptibility of very small samples, to analyse tiny magnetic signals and, in some cases, even to accurately map Fermi surfaces ${ }^{20-22}$.

Our set-up measured $\tau$, the component of $\tau$ along the rotation axis of a $25-\mu \mathrm{m}$ - or $50-\mu \mathrm{m}$-thick cantilever, with the sample glued to the cantilever tip. $H$ was applied at a tilt angle $\varphi$ with respect to the $c$ axis (perpendicular to the interface). The cantilever deflection was detected capacitively. The moment $m$ is given by $m=\tau /\left(\mu_{0} H \sin \theta\right)$, where $\mu_{0}$ is the vacuum permeability and $\theta$ is the angle between $m$ and $H$ (with $m$ in plane, $\theta=90^{\circ}-\varphi$; see the discussion below). We used the measured angular dependence of the zero-field capacitance of the cantilever set-up to calibrate the spring constant of the cantilever. Knowing the spring constant, we quantitatively determine the value of $m$. The cantilever set-up can resolve changes ${ }^{22}$ in $m$ of $\delta m=10^{-13}-10^{-12} \mathrm{~A} \mathrm{~m}^{2}$ at $10 \mathrm{~T}$.

All samples investigated were grown using nominally identical parameters for the substrate preparation and the pulsed laser deposition. The films were patterned with $\mathrm{Nb}$ Ohmic contacts using photolithography and painted with silver paste on the back. The only intended difference between the samples is that for one reference sample (named the '0 u.c.' sample) a shutter in front of the substrate was used to block the growth of LAO (Fig. 1a). The resistance of the interface samples was measured using the $\mathrm{Nb}$ Ohmic contacts. The LAO/STO interfaces were found to be superconducting below $120 \mathrm{mK}$. The superconducting temperature is slightly lower than that of many other LAO/STO samples grown

\footnotetext{
${ }^{1}$ Department of Physics, Massachusetts Institute of Technology, Cambridge, Massachusetts 02139, USA, ${ }^{2}$ Center for Electronic Correlations and Magnetism, University of Augsburg, 86135 Augsburg, Germany, ${ }^{3}$ Max Planck Institute for Solid State Research, 70569 Stuttgart, Germany. *e-mail: ashoori@mit.edu.
} 
a

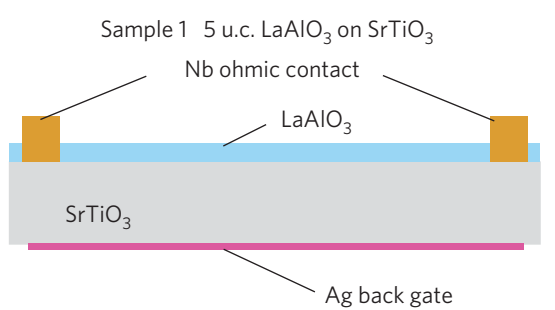

Sample 2 u u.c. $\mathrm{LaAlO}_{3}$ on $\mathrm{SrTiO}_{3}$

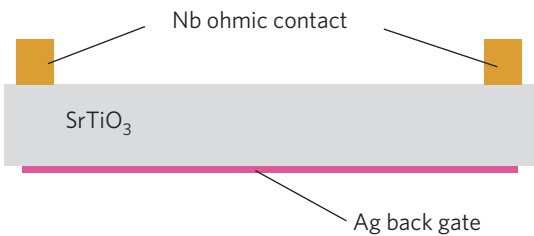

C

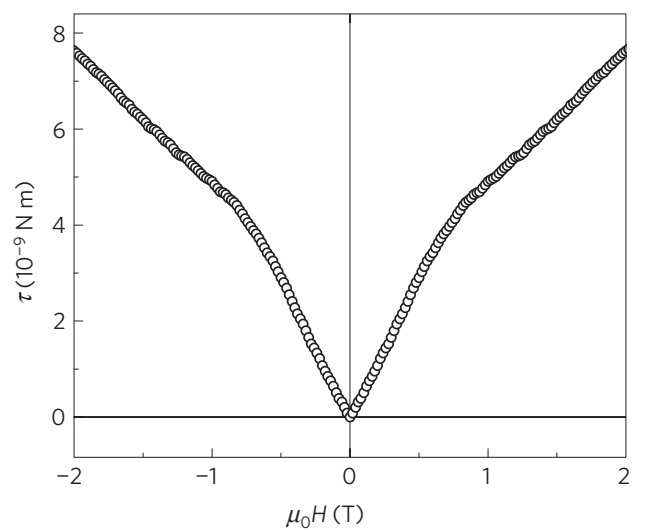

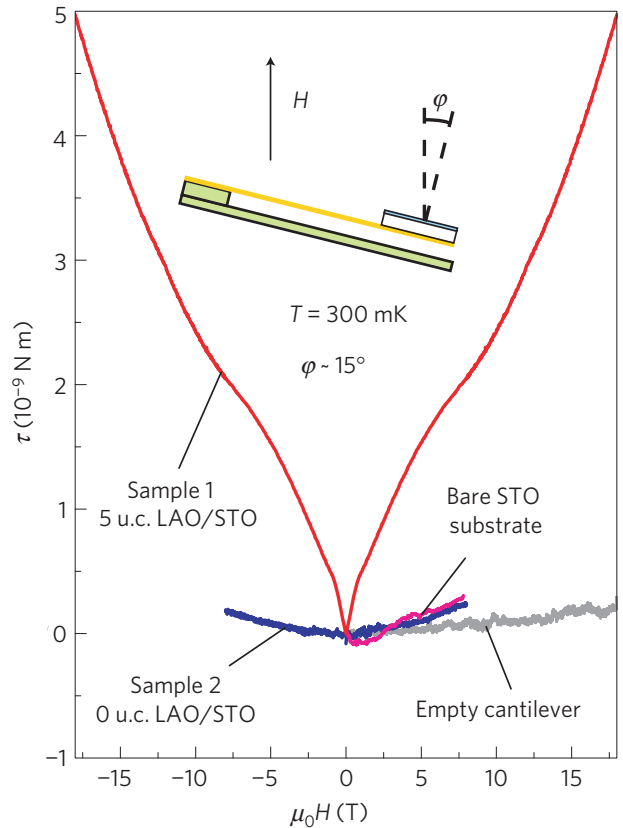

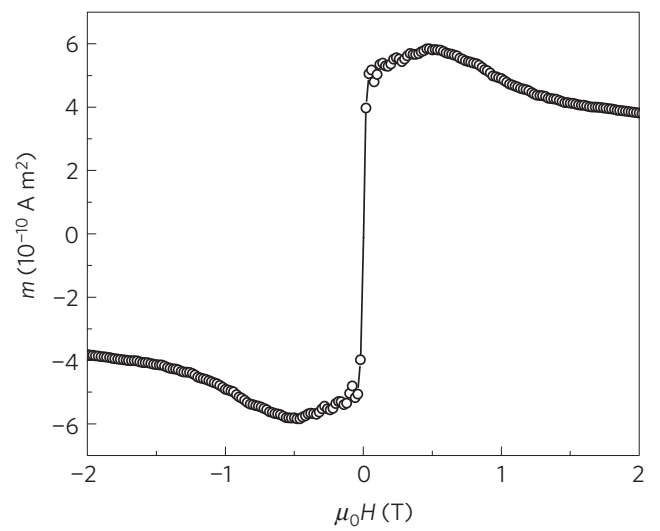

Figure 1| Torque magnetometry of LAO/STO oxide interface. a, A schematic representation of an interface sample (sample 1) and of a 0 u.c. background sample (sample 2), which were grown in the same conditions. b. The field dependence of the torque curves of various test samples (cantilever only, bare STO substrate and the 0 u.c. sample) and a interface sample, taken at $T=300 \mathrm{mK}$ and tilt angle $\varphi \sim 15^{\circ}$. Inset: A schematic representation of the cantilever set-up. c, In sample 1, the field dependence of the torque curve is linear and symmetric below $0.5 \mathrm{~T}$. $\mathbf{d}$, In sample 1 , the magnetic moment $m$ jumps to a finite value within milliteslas near zero field.

in the same conditions, which might be the result of unintended variations of growth parameters.

An example of the $\tau-H$ dependence is shown as the red curve in Fig. $1 \mathrm{~b}$ for a 5 u.c. sample. The torque signal has a pronounced reversible curve with a sharp 'cusp' at low field. This cusp is displayed clearly by Fig. 1c, which zooms into this cusp. Figure 1d shows $m$ determined from the $\tau-H$ curve at $-2 \mathrm{~T} \leq \mu_{0} H \leq 2 \mathrm{~T}$. The $\mathrm{V}$ shape of the $\tau-H$ curve centred at $H=0$ yields a non-zero, $H$-independent $m$ for $\mu_{0} H$ up to $0.5 \mathrm{~T}$. Close to $H=0, m$ jumps to $5 \times 10^{-10} \mathrm{~A} \mathrm{~m}^{2}$, corresponding to $0.3 \sim 0.4 \mu_{\mathrm{B}}$ per interface unit cell (assuming that the signal is generated by the STO unit cell next to the interface, see below). The values of $m$ very close to zero field $\left(\left|\mu_{0} \mathrm{H}\right| \leq 5 \mathrm{mT}\right)$ are hard to determine, because the small $H$ causes a large relative noise in $m$. At $\left|\mu_{0} H\right|=5 \mathrm{mT}, \delta m \sim 4 \times 10^{-10} \mathrm{~A} \mathrm{~m}^{2}$, which is close to the magnitude of $m$. Starting at fields of order $1 \mathrm{~T}, m$ diminishes gradually at higher $H$, suggesting that an extra contribution appears in high fields. This high-field contribution was found to vary among different runs. Below we focus on the low-field behaviour.

To explore whether the torque signals originate from the LAO/STO interface, we carried out control experiments using reference samples. Sizable torque signals were only observed from samples containing LAO/STO interfaces, the torque of which exceeds that of all background samples by two orders of magnitude (Fig. 1b). In particular, the superconducting $\mathrm{Nb}$ Ohmic contacts are unlikely to be the source of the torque signal, as the torque is found far above the upper critical field of $\mathrm{Nb}(0.4 \mathrm{~T}$ for bulk or $2 \mathrm{~T}$ for thin films at $0 \mathrm{~K})$. Moreover, all background $m$ will be oriented closely parallel to $H$, thus creating small torque responses only. The background $m$ is also proportional to $H$, as these materials are paramagnetic or diamagnetic. Furthermore, we measured a 5-u.c.-thick LAO film grown on an LAO substrate. The torque signal is again two orders of magnitude smaller than that of the 5 u.c. LAO/STO sample, excluding the possible contribution from defects in the LAO film (see the Supplementary Information). We therefore conclude that the observed large torque indeed arises from the presence of the LAO/STO interface.

A chief motivation for our study was to determine whether the superconductivity and magnetic order appear simultaneously or exist as separate phases in the temperature $T-H$ phase diagram. We observe that below the superconducting transition temperature $T_{\mathrm{c}}$, the magnetic ordering signal and the superconducting state 
a

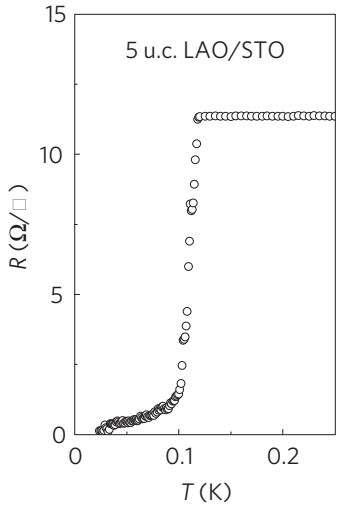

b
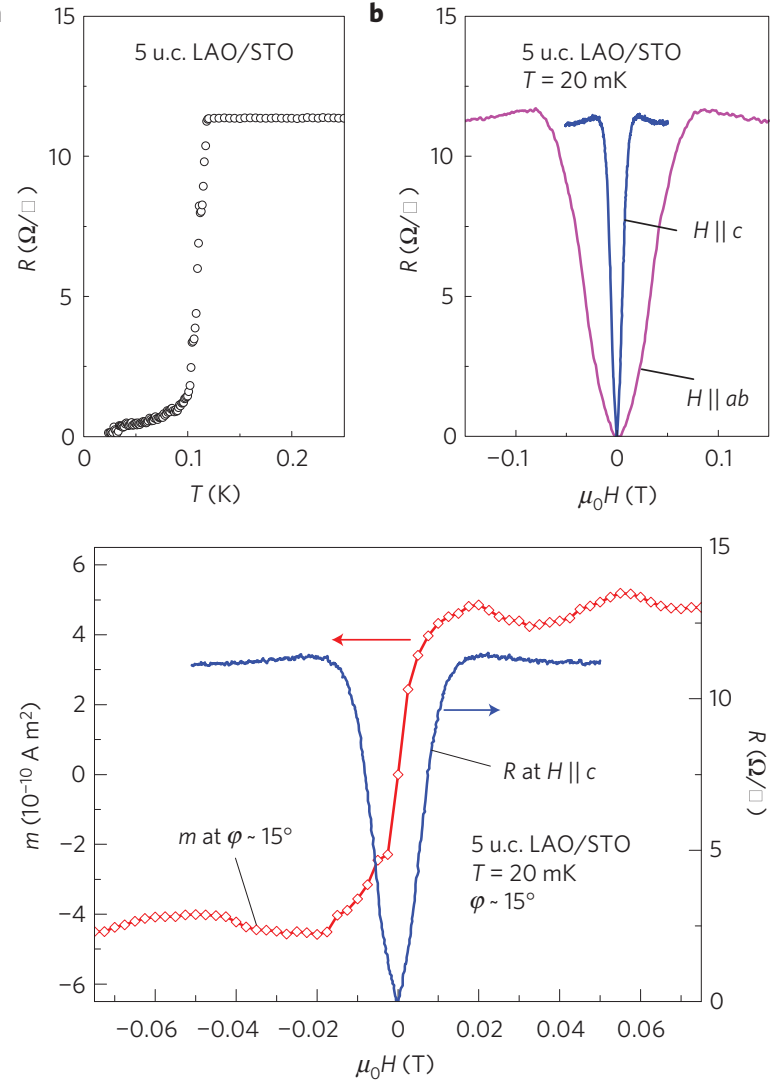

Figure $\mathbf{2}$ | Coexistence of superconductivity and magnetic ordering in a 5 u.c. LAO/STO interface sample. a, The $T$ dependence of the resistance $R$ shows a superconducting transition at $T_{\mathrm{c}}=120 \mathrm{mK}$. $\mathbf{b}, H$ dependence of $R$ in different field directions taken at $T=20 \mathrm{mK}$. $\mathbf{c}, \mathrm{H}$ dependence of $m$ measured at $T=20 \mathrm{mK}$ at tilt angle $\varphi \sim 15^{\circ}$ away from the $c$ axis. The $R-H$ curve is also plotted with $H$ parallel to the $c$ axis.

coexist. For the sample of Fig. 2, for example, the superconducting transition occurs at $120 \mathrm{mK}$ at $H=0$, with a resistance foot extending to $25 \mathrm{mK}$. The $R-H$ curves measured at $20 \mathrm{mK}$ with
$H$ parallel and perpendicular to the interface plane are plotted in Fig. 2b. While the interface is superconducting, the $m-H$ curve at $20 \mathrm{mK}$ displays the same jump at small fields (Fig. 2c) as observed at higher temperatures (Fig. 1d). Notably, a finite $m$ is recorded at $\mu_{0} H \sim 5 \mathrm{mT}$, whereas the sample resistance $R$ does not reach the normal-state value until $\mu_{0} H \sim 20 \mathrm{mT}$. The magnetic ordering signal and the superconducting state are therefore found to coexist.

The magnetic ordering signal is robust at elevated temperatures. For the 5 u.c. LAO/STO sample, $m$ does not show significant temperature dependence even up to $40 \mathrm{~K}$ (Fig. 3), the highest $T$ at which this sample was investigated. In another 5 u.c. LAO/STO sample, $m$ was found to be non-zero up to $200 \mathrm{~K}$ (see the Supplementary Information). Such $T$ dependence is consistent with previous results ${ }^{12}$ reporting the existence of an ordering state at room temperature. The high magnetic ordering temperature indicates a strong magnetic exchange coupling.

The magnetic-field dependence of $m$ can be described by the Langevin function characteristic for superparamagnetism, where spins are aligned in small-size domains to behave as large classical magnetic moments ${ }^{23}$. However, superparamagnetic samples usually show a strong temperature dependence in the low-field $m-H$ curves, a feature missing from the $m-H$ curves in Fig. 3. Noise in our measurements of $m$ at fields close to zero may obscure this feature. Because $m$ saturates at about $30 \mathrm{mT}$ at $T$ up to at least $40 \mathrm{~K}$, the lower bound of the collective classical moment is around $10^{3} \mu_{\mathrm{B}}$. On the other hand, the $m-H$ curves are also consistent with a very soft ferromagnet whose hysteresis loop is hidden by the $m$ noise at $\left|\mu_{0} H\right|<5 \mathrm{mT}$. Although these two possibilities cannot be distinguished by our data, both of them suggest a strong ferromagnetic-like magnetic coupling within domains.

To determine the orientation of the magnetic moment, we made a series of torque measurements in which the sample tilt angle was varied (see the inset of Fig. 1b). Because $\boldsymbol{\tau}=\mathbf{m} \times \mathbf{B}=\mu_{0} m H_{\perp}$, where $H_{\perp}$ is the component of $H$ perpendicular to $\mathbf{m}$, the orientation of the moments can be discerned by tracking the angular dependence of the torque signal. In a highly anisotropic system, $m$ is determined by $H_{\|}$, the field component parallel to $m$. Thus if $H_{\|}$is large enough to saturate $m, \tau$ will increase as a sine function of the angle between $H$ and $m$. On the other hand, once $H_{\|}$is insufficient to saturate $m$, $\tau$ will stop following the sine behaviour.
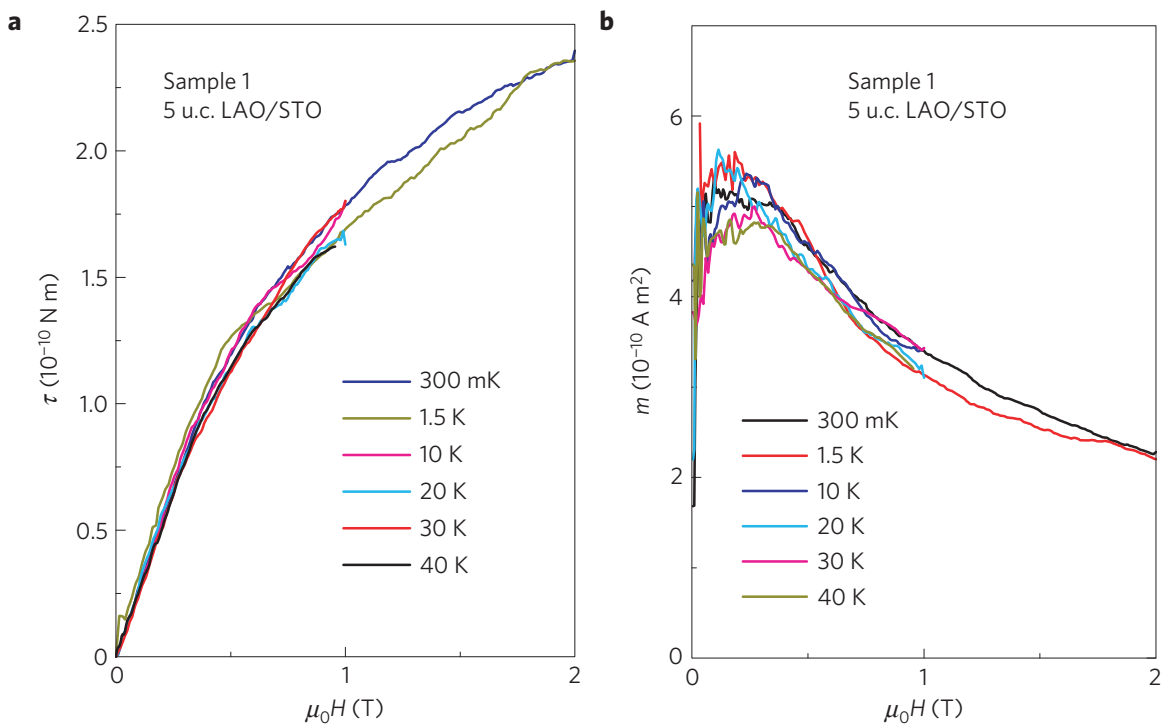

Figure 3 | Magnetic ordering persisting to elevated temperature. a, Torque versus $H$ curves of the 5 u.c. LAO/STO sample measured at selected $T$ between $300 \mathrm{mK}$ and $40 \mathrm{~K}$. Within the measurement noise, no strong temperature dependence is observed. The tilt angle is about $49^{\circ}$. $\mathbf{b}$, The curves of $m$ versus $\mathrm{H}$ calculated from the torque curves. 


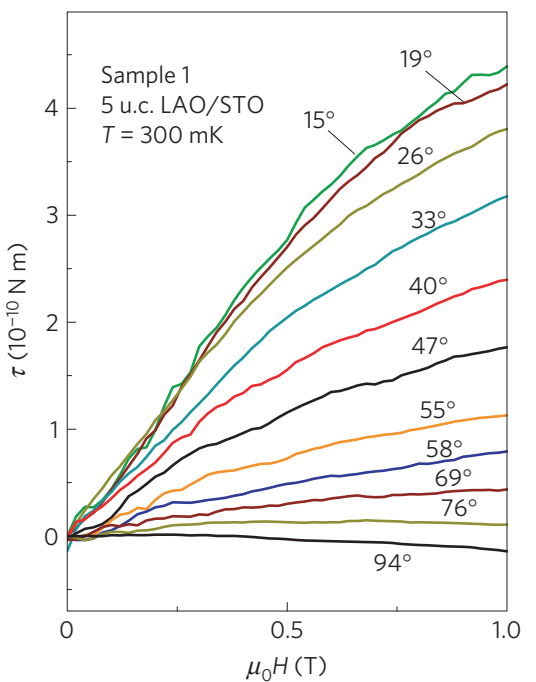

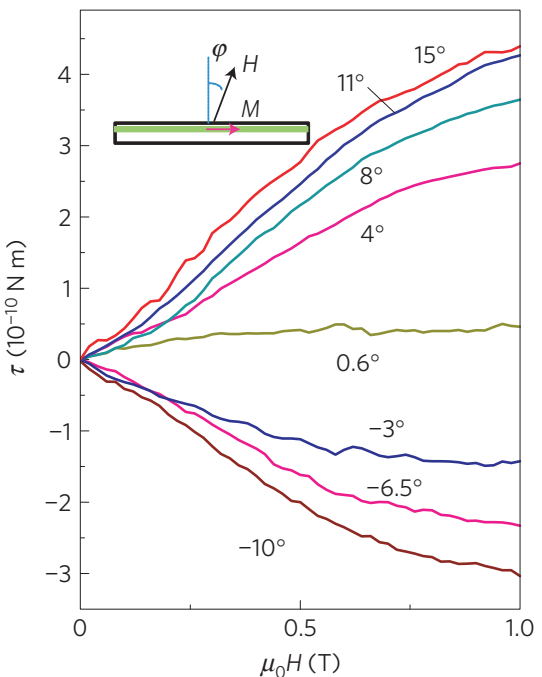

Figure 4 | Angular dependence of the interface torque indicating an in-plane saturation magnetic moment. $\mathbf{a}, \mathbf{b}$, At $T=300 \mathrm{mK}$, the magnetic torque of the 5 u.c. LAO/STO sample is measured at various tilt angles $\varphi$ between $15^{\circ}$ and $94^{\circ}$ (a) and between $-10^{\circ}$ and $15^{\circ}$ (b). The inset of $\mathbf{b}$ shows the geometry of the field $H$ and magnetization $M$, and the definition of the tilt angle $\varphi$.

The angle dependence shows that the saturation magnetic moment stays in the plane of the interface. We carried out low-field torque measurements at $300 \mathrm{mK}$ at 30 different tilt angles. Figure 4 shows the $\tau-H$ curves at several selected angles $\varphi$. As shown in Fig. 4 a, as $\varphi$ changes from $15^{\circ}$ to $94^{\circ}, \tau$ decreases monotonically and slowly approaches zero at $\varphi \sim 90^{\circ}$, where $\mathbf{H}$ is almost parallel to $\mathbf{m}$. On the other hand, as $\varphi$ varies between $+15^{\circ}$ and $-10^{\circ}, \mathbf{H}$ is almost perpendicular to $\mathbf{m}$. $H_{\|}$decreases and eventually changes to the opposite direction. The in-plane magnetic moment drops to zero once $H_{\|}$is close to zero. As a result, the $\tau(H)$ curves swing from a positive saturation at $\varphi \sim 15^{\circ}$ to a negative saturation at $\varphi \sim-10^{\circ}$.

Our data show that two-dimensional superconductivity and magnetic order coexist at n-type LAO/STO interfaces. The results leave open the question of whether the same electrons are generating the superconducting and the magnetic order. The measured results can be accounted for by scenarios of spatial phase separation, in which inhomogeneous magnetic and superconducting electron layers are generated either in different lateral puddles, or at different depths from the interface. One possible cause of such inhomogeneities is a non-uniform distribution of possible oxygen vacancies in the STO. This notion is in accord with a proposal that the oxygen vacancies in the interfacial $\mathrm{TiO}_{2}$ layers stabilize ferromagnetic-type order of the $\mathrm{Ti}$ ions close to the interface, as supported by density functional theory calculation ${ }^{24}$. In this scenario the superconducting phase is in close contact with the ferromagnetic phase, so the superconducting phase is affected by the ferromagnetism. Furthermore, the data are also consistent with the idea that the same electron system forms a magnetically ordered, superconducting electron liquid.

We note that, after our submission of the manuscript $\mathrm{t}^{25}$, two experiments were reported to support the coexistence of superconductivity and ferromagnetism at the LAO/STO interface, based on hysteretic magnetoresistance ${ }^{26,27}$ and scanning superconducting quantum interference device imaging ${ }^{28}$.

In conclusion, using torque magnetometry we have made quantitative measurements of the magnetic moment of LAO/STO interfaces in a wide range of magnetic field and temperature, directly showing the presence of magnetic order in the twodimensional electron liquid of LAO/STO interfaces. The order is characterized by a superparamagnetic-like behaviour, with saturation magnetic moments of $\sim 0.3 \mu_{\mathrm{B}}$ per interface unit cell oriented in plane, persisting beyond $200 \mathrm{~K}$. Below $120 \mathrm{mK}$, the ferromagnetic-like magnetic order and the two-dimensional superconductivity coexist.

\section{Methods}

The LAO/STO heterostructures were grown at the University of Augsburg using pulsed-laser deposition with in situ monitoring of the LAO layer thickness by reflection high-energy electron diffraction. The single-crystalline STO substrates were $\mathrm{TiO}_{2}$ terminated. Their lateral size was $5 \times 5 \mathrm{~mm}^{2}$ and their thickness was $1 \mathrm{~mm}$. The LAO layers were grown at an oxygen pressure of $8 \times 10^{-5} \mathrm{mbar}$ at $780^{\circ} \mathrm{C}$ to a thickness of 5 u.c with a subsequent cooldown to $300 \mathrm{~K}$ in 0.5 bar of oxygen. The sputtered Ohmic $\mathrm{Nb}$ contacts filled holes patterned by etching with an $\mathrm{Ar}$ ion beam. The reference $(0$ u.c.) samples were grown in the same conditions (oxygen pressure of $8 \times 10^{-5} \mathrm{mbar}$ at $780^{\circ} \mathrm{C}$ ).

The magnetization measurements were made with a home-built cantilever-based torque magnetometry apparatus at MIT. Cantilevers are made from thin gold or brass foils. We deposit gold film on a sapphire and put it under the cantilever. The torque is tracked by measuring the capacitance between the cantilever and the gold film, using a GR1615 capacitance bridge or an AH2700A capacitance bridge. To calibrate the spring constant of the cantilever, we rotate the cantilever set-up under zero magnetic field to measure the capacitance change caused by the weight of the sample wafer.

Received 3 May 2011; accepted 2 August 2011; published online 4 September 2011

\section{References}

1. Ohtomo, A. \& Hwang, H. Y. A high-mobility electron gas at the $\mathrm{LaAlO}_{3} / \mathrm{SrTiO}_{3}$ heterointerface. Nature 427, 423-426 (2004).

2. Thiel, S. et al. Tunable quasi-two dimensional electron gases in oxide heterostructures. Science 313, 1942-1945 (2006).

3. Reyren, N. et al. Superconducting interfaces between insulating oxides. Science 317, 1196-1199 (2007).

4. Brinkman, A. et al. Magnetic effects at the interface between non-magnetic oxides. Nature Mater. 6, 493-496 (2007).

5. Fulde, F. \& Ferrell, R. A. Superconductivity in a strong spin-exchange field. Phys. Rev. 135, A550-A563 (1964).

6. Larkin, A. I. \& Ovchinikov, Y. N. Inhomogenous state of superconductors. Sov. Phys. JETP. 20, 762 (1965).

7. Lynn, J. W. et al. Antiferromagnetic ordering of Ru and $\mathrm{Gd}$ in superconducting $\mathrm{RuSr}_{2} \mathrm{GdCu}_{2} \mathrm{O}_{8}$. Phys. Rev. B 61, 14964-14967 (2000).

8. Pickett, W. E. et al. Superconductivity in ferromagnetic $\mathrm{RuSr}_{2} \mathrm{GdCu}_{2} \mathrm{O}_{8}$. Phys. Rev. Lett. 83, 3713-3716 (1999).

9. Saxena, S. S. et al. Superconductivity at the border of itinerant electron ferromagnetism in $\mathrm{UGe}_{2}$. Nature 406, 587-592 (2000).

10. Shalom, M. B. et al. Anisotropic magnetotransport at the $\mathrm{SrTiO}_{3} / \mathrm{LaAlO}_{3}$ interface. Phys. Rev. B 80, 140403(R) (2009).

11. Seri, S. \& Klein, L. Antisymmetric magnetoresistance of the $\mathrm{SrTiO}_{3} / \mathrm{LaAlO}_{3}$ interface. Phys. Rev. B 80, 180410 (2009).

12. Ariando, et al. Electronic phase separation at the $\mathrm{LaAlO}_{3} / \mathrm{SrTiO}_{3}$ interface. Nature Comm. 2, 188-194 (2011). 
13. Okamoto, S., Millis, A. J. \& Spaldin, A. Lattice relaxation in oxide heterostructures: $\mathrm{LaAlO}_{3} / \mathrm{SrTiO}_{3}$ superlattices. Phys. Rev. Lett. 97, 056802 (2006).

14. Pentcheva, R. \& Pickett, W. E. Charge localization or itineracy at $\mathrm{LaAlO}_{3} / \mathrm{SrTiO}_{3}$ interfaces: Hole polarons, oxygen vacancies, and mobile electrons. Phys. Rev. B 74, 035112 (2006).

15. Janicka, K. et al. Magnetism of $\mathrm{LaAlO}_{3} / \mathrm{SrTiO}_{3}$ superlattices. J. Appl. Phys. 103, 07B508 (2008)

16. Zhong, Z. C. \& Kelly, P. J. Electronic-structure-induced reconstruction and magnetic ordering at the $\mathrm{LaAlO}_{3} \mid \mathrm{SrTiO}_{3}$ interface. Europhys. Lett. 84, 27001 (2008).

17. Pavlenko, N. \& Kopp, T. Structural relaxation and metal-insulator transition at the interface between $\mathrm{SrTiO}_{3}$ and $\mathrm{LaAlO}_{3}$. Surf. Sci. 605, 1114-1121 (2011).

18. Huijben, M. et al. Structure-property relation of $\mathrm{SrTiO}_{3} / \mathrm{LaAlO}_{3}$ interfaces. Adv. Mater. 21, 1665-1677 (2009).

19. Sachs, M. et al. Anomalous magneto-transport at the superconducting interface between $\mathrm{LaAlO}_{3}$ and $\mathrm{SrTiO}_{3}$. Physica C 470, 1-2 (2010).

20. Farrell, D. E. et al. Experimental evidence for a transverse magnetization of the Abrikosov lattice in anisotropic superconductors. Phys. Rev. Lett 61, 2805-2808 (1988).

21. Sebastian, S. E. et al. A multi-component Fermi surface in the vortex state of an underdoped high $T_{\mathrm{c}}$ superconductor. Nature 454, 200-203 (2008).

22. Li, L. et al. Low temperature vortex liquid in $\mathrm{La}_{2-x} \mathrm{Sr}_{x} \mathrm{CuO}_{4}$. Nature Phys. 3, 311-314 (2007).

23. Morrish, A. H. The Physical Principles of Magnetism (John Wiley, 1965).

24. Pavlenko, N., Kopp, T., Sawatzky, G. A. \& Mannhart, J. Magnetism and superconductivity at LAO/STO-interfaces both generated by the Ti $3 \mathrm{~d}$ interface electrons? Preprint at http://arxiv.org/abs/1105.1163.

25. Li, L., Richter, C., Mannhart, J. \& Ashoori, R. C. Direct magnetization measurement of the $\mathrm{LaAlO}_{3} / \mathrm{SrTiO}_{3}$ heterostructure. Abstract \#A34.009 APS March Meeting 2011, American Physical Society, 21-25 March (2011).
26. Dikin, et al. Coexistence of superconductivity and ferromagnetism in two dimensions. Phys. Rev. Lett. 107, 056802 (2011).

27. Mehta, M. et al. Hysteretic magneto-resistance at the $\mathrm{LaAlO}_{3}-\mathrm{SrTiO}_{3}$ interface-interplay between superconducting and ferromagnetic properties. Abstract \#A34.012 APS March Meeting 2011, American Physical Society, 21-25 March (2011).

28. Bert, J. A. et al. Direct imaging of the coexistence of ferromagnetism and suprconductivity at the $\mathrm{LaAlO}_{3} / \mathrm{SrTiO}_{3}$ interface. Nature Phys. 7, 767-771 (2011).

\section{Acknowledgements}

The authors acknowledge discussions with D. Grundler, T. Kopp, P. A. Lee and G. A. Sawatzky, and experimental assistance from T. Murphy, J-H. Park and S. Hannahs. This work was supported by the Army Research Office (54173PH), by the Deutsche Forschungsgemeinschaft (TRR 80) and by the EC (OxIDes). L.L. would like to thank the MIT Pappalardo Fellowships in Physics for their support. The high-field experiments were performed at the National High Magnetic Field Laboratory, which is supported by National Science Foundation Cooperative Agreement DMR-084173, by the State of Florida and by the DOE.

\section{Author contributions}

The studies were designed, planned and analysed by all authors, who also wrote the manuscript. C.R. grew the samples; L.L. carried out the torque and resistivity measurements and the data analysis.

\section{Additional information}

The authors declare no competing financial interests. Supplementary information accompanies this paper on www.nature.com/naturephysics. Reprints and permissions information is available online at http://www.nature.com/reprints. Correspondence and requests for materials should be addressed to R.C.A. 